\title{
Hierarchical Microstructure Evolution of Yb-filled CoSb3 Skutterudite Obtained by Spark Plasma Sintering
}

Paula Rebeca Realyvazquez-Guevara ${ }^{1}$, Maria Cristina Grijalva-Castillo ${ }^{2}$, Alejandro Faudoa-Arzate ${ }^{1}$, Carlos Arzate-Quintana ${ }^{3}$, H. J. Morales-Rodriguez ${ }^{4}$ and Jose Andres Matutes-Aquino ${ }^{2}$

${ }^{1}$ Tecnológico de Monterrey, Chihuahua, Chihuahua, Mexico, ${ }^{2}$ Centro de Investigacion en Materiales Avanzados, S.C., Chihuahua, Chihuahua, Mexico, ${ }^{3}$ Universidad Autónoma de Chihuahua, Chihuahua, Chihuahua, Mexico, ${ }^{4}$ Universidad Tecnológica de Chihuahua Sur, Chihuahua, Chihuahua, Mexico

Skutterudite antimonides are promising candidates for the next-generation of thermoelectric (TE) materials of electrical power generation devices for converting waste heat into electricity and for thermal cooling [1]. The performance of a TE material is related to the dimensionless thermoelectric figure of merit, ZT, defined as $Z T=S^{2} \sigma T /\left(\kappa_{e}+\kappa_{L}\right)$, where $S$ is the Seebeck coefficient, $T$ is the absolute temperature, $\sigma$ is the electrical conductivity, $\kappa_{e}$ and $\kappa_{L}$ are the electronic and lattice thermal conductivities, respectively. Various approaches have been taken to improve ZT value, for instance, thermal conductivity can be lowered by filling the voids of the crystal structure, nanostructuring [2] and the formation of hierarchical structures, which significantly reduce the lattice thermal conductivity through all-scale length phonon scattering [3].

Polycrystalline hierarchically structured $\mathrm{Yb}$-filled skutterudite $\left(\mathrm{CoSb}_{3}\right)$ was prepared by the meltingquenching-annealing-sintering method [4] [5] Phases identification was carried out by powder X-ray

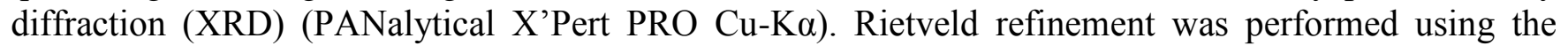
Fullprof program [6]. The morphology of the sample was analyzed by scanning electron microscopy (SEM) (JEOL-JSM 5800). Chemical composition and sample homogeneity were checked by EnergyDispersive X-Ray Spectrometry (EDS) microanalysis attached to the SEM.

Stoichiometric amounts of Yb powder with a purity of $99.9 \%$ (Alfa Aesar), Co powder with a purity of 99.998\% (Alfa Aesar) and Sb powder with a purity of $99.999 \%$ (Alfa Aesar) were loaded into a vacuum sealed quartz ampoule. As a first step, the content of the ampule was melted at $1100{ }^{\circ} \mathrm{C}$ and kept at this temperature during 24 hours. Then, the ampoule was quenched in a supersaturated salt solution of $\mathrm{NaCl}$ in water. Afterwards, the sample was annealed at $650{ }^{\circ} \mathrm{C}$ during 120 hours, to promote the skutterudite phase formation. Subsequently, the sample was re-melted under vacuum in an induction furnace and the melt was ejected onto the copper wheel of a melt-spinning system, rotating with a tangential velocity of $30 \mathrm{~m} / \mathrm{s}$. The microstructure of the obtained ribbons is shown in Figure 1. The (a) free surface is composed by grain agglomerates with a grain size distribution from 0.25 to $1 \mathrm{~mm}$. The (b) contact surface are smaller in size, which corresponds to a higher solidification rate. The variation of the grain size along the thickness and the average thickness of the ribbons $(28.11 \mathrm{~mm})$ can be observed in Figure 1(c). Ribbons are composed by several crystalline phases: $\mathrm{Sb}, \mathrm{CoSb}, \mathrm{CoSb} 2$ and $\mathrm{CoSb}_{3}$; as shown in Figure 2(a). The ribbons were grounded and the resulting powder was synthesized by spark plasma sintering (SPS) under a pressure of $30 \mathrm{MPa}$ at $650{ }^{\circ} \mathrm{C}$ for 5 minutes. The SEM image of the freshly fractured surface, Figure 1(d), shows the presence of well-defined grains with smooth surface -characteristic of brittle failure - with a wide grain size distribution, containing grains with a size less than $100 \mathrm{~nm}$ - as shown in Figure 1(d) - to $2 \mathrm{~mm}$. The actual composition was determined by averaging random spots on the surface of the sample and by normalizing the resulting chemical formula to four cobalt atoms. A nominal composition of 
Yb0.29 $\mathrm{Co}_{4} \mathrm{Sb}_{11.01}$ was determined. Figure 2(b) shows the Rietveld refinement using FullProf software. A single-phase sample with a lattice parameter equals to $9.0444 \AA$ was determined.

Thermal conductivity was measured on $14 \times 2 \times 2 \mathrm{~mm}^{3}$ bar samples using the thermal transport option (TTO) of a Physical Property Measurement System (PPMS, Quantum Design). The measured total thermal conductivity, $\kappa_{\mathrm{T}}$, at room temperature was $2.72 \mathrm{~W} / \mathrm{K}-\mathrm{m}$ and $2.3 \mathrm{~W} / \mathrm{K}-\mathrm{m}$ at $400 \mathrm{~K}$ (the highest measurement temperature available), which is one of the lowest measured values for this compound, as reported in the literature [7].
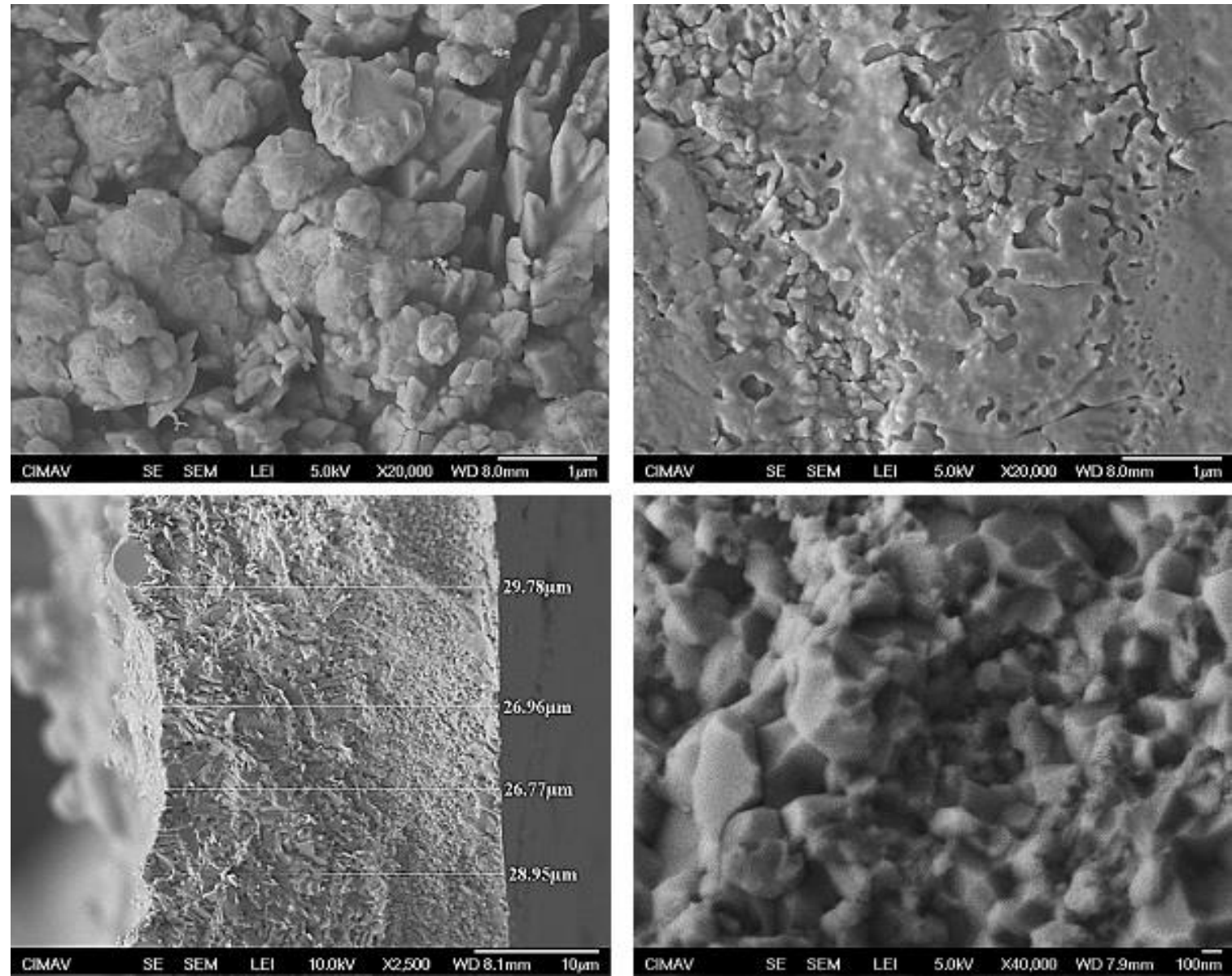

Figure 1. Microstructure evolution of the Yb-filled skutterudite: (a) Free surface of the melt spun ribbon, (b) Contact surface of the ribbon, (c) Transversal view of the ribbon and (d) hierarchically microstructure obtained after Spark Plasma Sintering. 

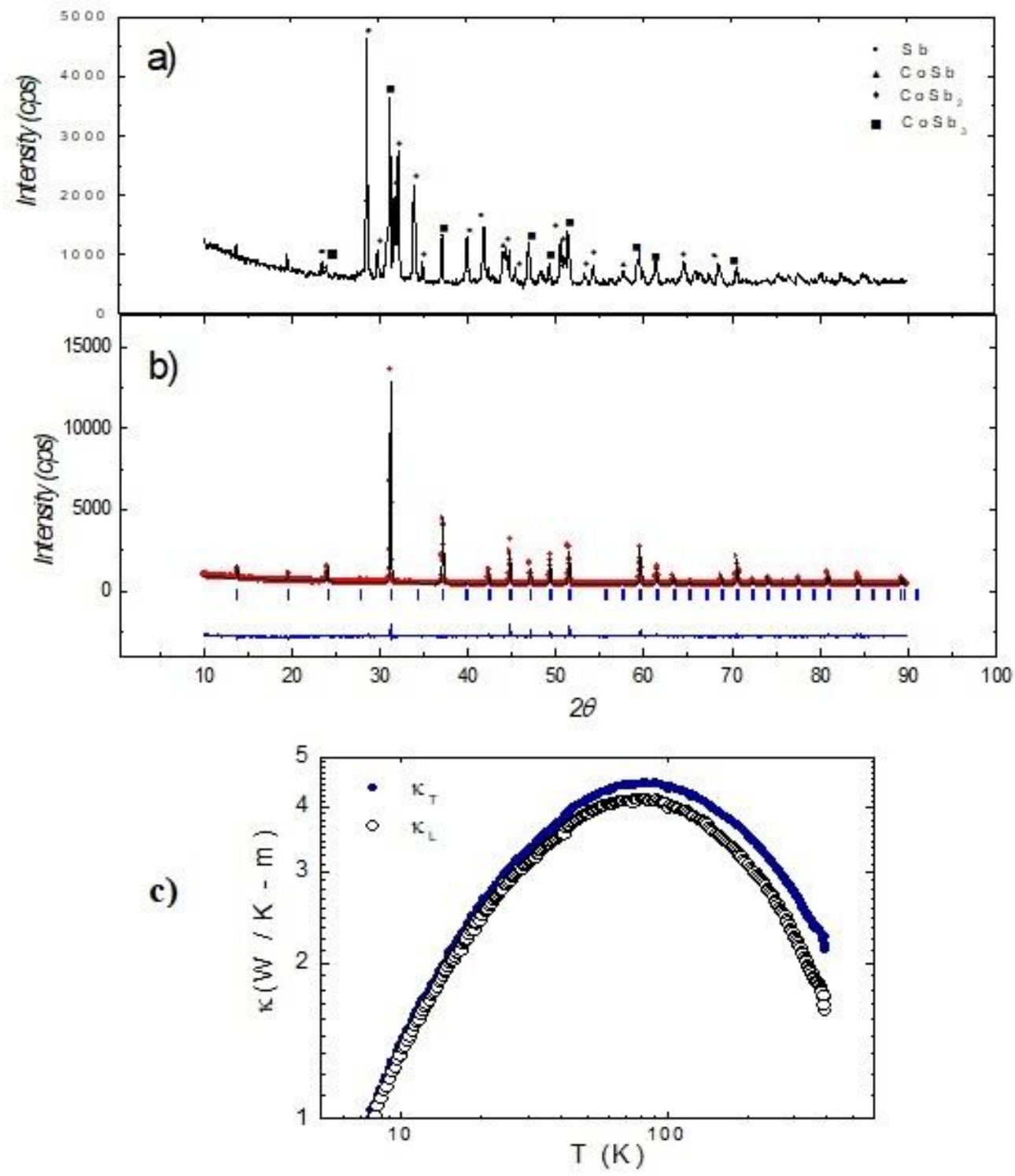

Figure 2. (a) XRD patterns of the melt-spun ribbons and (b) Plot of the observed intensity, the calculated intensity and the intensity difference for the Rietveld refinement of the sample. A single-phase filled skutterudite sample with a lattice parameter equals to $9.0444 \AA$ was determined. (c) Total thermal conductivity $(\kappa \mathrm{T})$ and calculated lattice thermal contribution $(\kappa \mathrm{L})$, measured between 3 and $400 \mathrm{~K}$.

\section{References}

[1] W. Li, J. Wang, Y. Xie, J. L. Gray, J. J. Heremans, H. B. Kang, B. Poudel, S. T. Huxtable, and S. Priya, Chemistry of Materials 31, 862 (2019).

[2] R. Swetha, M. U. Kumar, and L. Kumari, in AIP Conference Proceedings (AIP Publishing LLC, 2019), p. 020104.

[3] N. Liu, S. E. Rezaei, W. A. Jensen, S. Song, Z. Ren, K. Esfarjani, M. Zebarjadi, and J. A. Floro, Advanced Functional Materials 29, 1903157 (2019). [4] P. Qiu, X. Shi, X. Chen, X. Huang, R. Liu, and L. Chen, Journal of Alloys and Compounds 509, 1101 (2011).

[5] K. H. Lee, S. H. Bae, and S.-M. Choi, Materials 13, 87 (2020). 
[6] J. Rodríguez-Carvajal, Physica B: Condensed Matter 192, 55 (1993).

[7] Z. Zhou et al., Journal of Materiomics 5, 702 (2019). 